\title{
Satisfação e insatisfação da equipe de enfermagem em unidades de urgência e emergência: revisão integrativa
}

RESUMO | Objetivo: Descrever, a partir da literatura publicada no período entre 2015 e 2020, a satisfação e insatisfação da equipe de enfermagem no contexto de trabalho em unidades de urgência e emergência. Metodologia: Estudo do tipo Revisão Integrativa, realizada nas bases de dados MEDLINE, LILACS, SciELO e BVS. A amostra inicial foi de 156 artigos encontrados nas bases de dados, destes apenas sete foram selecionados segundo os critérios estabelecidos. Para tratamento dos dados, utilizou-se a análise de conteúdo. Resultados: Emergiram duas categorias temáticas: Fatores de satisfação no trabalho de enfermagem em urgência e emergência e Fatores de insatisfação no trabalho de enfermagem em urgência e emergência. Conclusão: A satisfação está relacionada a condições de trabalho; realização e reconhecimento profissional; remuneração e formação continuada. Já a insatisfação, está relacionada a falta de interação entre a equipe; falta de reconhecimento profissional, apoio e autonomia; e condições de trabalho inadequadas, como a sobrecarga.

Palavras-chaves: Satisfação no emprego; Enfermagem; Enfermeiras e enfermeiros; Serviços médicos de emergência.

\begin{abstract}
Objective: To describe, from the literature published between 2015 and 2020, the satisfaction and dissatisfaction of the nursing team in the context of work in urgent and emergency units. Methodology: Integrative Review study, carried out in MEDLINE, LILACS, SciELO and BVS databases. The initial sample was 156 articles found in the databases, of which only seven were selected according to the established criteria. Content analysis was used to process the data. Results: Two thematic categories emerged: Factors of satisfaction in nursing work in urgency and emergency and Factors of dissatisfaction in nursing work in urgency and emergency. Conclusion: Satisfaction is related to working conditions; professional achievement and recognition; remuneration and continuing training. Dissatisfaction, on the other hand, is related to the lack of interaction between the team; lack of professional recognition, support and autonomy; and inadequate working conditions, such as overload.
\end{abstract}

Keywords: Job satisfaction; Nursing; Nurses; Emergency medical services.

RESUMEN | Objetivo: Describir, a partir de la literatura publicada entre 2015 y 2020, la satisfacción e insatisfacción del equipo de enfermería en el contexto del trabajo en unidades de urgencia y emergencia. Metodología: Estudio de revisión integrativa, realizado en bases de datos MEDLINE, LILACS, SciELO y BVS. La muestra inicial fue de 156 artículos encontrados en las bases de datos, de los cuales sólo siete fueron seleccionados de acuerdo con los criterios establecidos. El análisis de contenido se utilizó para procesar los datos. Resultados: Surgieron dos categorías temáticas: Factores de satisfacción en el trabajo de enfermería en urgencia y emergencia y Factores de insatisfacción en el trabajo de enfermería en urgencia y emergencia. Conclusión: La satisfacción está relacionada con las condiciones de trabajo; logro profesional y reconocimiento; remuneración y formación continua. La insatisfacción, por otro lado, está relacionada con la falta de interacción entre el equipo; falta de reconocimiento profesional, apoyo y autonomía; y condiciones de trabajo inadecuadas, como la sobrecarga.

Palabras claves: Satisfacción en el trabajo; Enfermería; Enfermeras y enfermeros; Servicios médicos de urgencia.

\section{Ana Gracinda Ignacio da Silva}

Enfermeira. Doutora em Enfermagem Universidade Federal do Rio de Janeiro (UFRJ). Docente Centro Universitário Metropolitano da Amazônia (UNIFAMAZ). Belém, PA.

ORCID: 0000-0001-6439-7842

\section{Sara Elene da Silva Mendonça}

Enfermeira. Discente Centro Universitário Metropolitano da Amazônia (UNIFAMAZ). Belém, PA.

ORCID: 0000-0003-4780-9774

\section{Andreza Cassundé Moraes}

Enfermeira. Discente Centro Universitário Metropolitano da Amazônia (UNIFAMAZ). Belém, PA.

ORCID: 0000-0001-7533-5700
Tatiane de Souza Vasconcelos

Enfermeira. Discente Centro Universitário Metropolitano da Amazônia (UNIFAMAZ). Belém, PA. ORCID: 0000-0003-2571-5101

\section{Gabriel Fazzi Costa}

Enfermeiro. Mestrando em Biologia Parasitária na Amazônia Universidade do Estado do Pará (UEPA)/Instituto Evandro Chagas (IEC/SVS). Belém, PA.

ORCID: 0000-0002-9672-5676

Recebido em: 19/03/2021

Aprovado em: 05/04/2021
INTRODUÇÃO

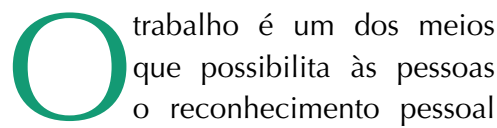
e profissional, assim sendo, a partir da função que ocupa o indivíduo busca a satisfação (pessoal e laboral) com a finalidade de assegurar qualidade de vida ${ }^{1}$. Considerando o trabalho da enfermagem, essa satisfação é importante, pois são profissionais que estão na linha de frente do cuidado com os pacientes e seus familiares, dessa forma, estar em um "estado emocionalmente positivo" contribui para a qualidade assistencial e profissional, e alcança expectativas e necessidades. 
Para que essa satisfação ocorra, os profissionais, em sua maioria, buscam por melhores remunerações, segurança no emprego, boas relações interpessoais no ambiente laborativo, valorização e reconhecimento ${ }^{2}$.

A satisfação no desenvolvimento das atividades laborais desenvolvidas pelos enfermeiros, está associada à manutenção da saúde do trabalhador e torna-se sinônimo, também, de qualidade de vida, pois contribui para o desenvolvimento de menores índices de adoecimento físico e mental ${ }^{3}$. Essa satisfação é um desafio, quando esse trabalho ocorre no contexto de trabalho em Unidades de Urgência e Emergência (UUE), que é caracterizado pelo atendimento aos usuários em situação grave, com ou sem risco de morte iminente ${ }^{4}$. Em face dessas atribuições, alguns impasses para prestar uma assistência de qualidade podem ser encontrados tanto para instituições, quanto para gestores e profissionais, como por exemplo, a sobrecarga da equipe de enfermagem e maior demanda de atividades, se comparada à capacidade e estrutura dos serviços $^{5}$, além da exposição a fatores físicos e psicológicos que afetam a qualidade do atendimento prestado e a saúde dos trabalhadores ${ }^{6}$.

Aliado a esses aspectos, durante as práticas curriculares, observaram-se queixas constantes de problemas de saúde e insatisfação pelos profissionais de enfermagem da unidade de urgência e emergência, o que trouxe interesse sobre a problemática a partir da seguinte questão norteadora: Quais evidências científicas presentes na literatura, publicada nos últimos cinco anos, representam a satisfação e insatisfação da equipe de enfermagem no contexto de trabalho de urgência e emergência?

O tema é relevante, uma vez que a enfermagem é o principal vínculo entre os usuários e o estabelecimento de saúde, pois presta um cuidado ininterrupto com os pacientes, tendo sob sua responsabilidade a manutenção, a recuperação e a reabilitação da saúde dos usuários por meio do cuidado. Desta forma, os cuidados prestados pela enfermagem refletirão em uma imagem positiva ou negativa da instituição de saúde, visto que, a satisfação dos usuários é definida a partir da experiência de se utilizar um serviço de saúde de acordo com as suas expectativas, definindo-se pelas avaliações positivas ou negativas das dimensões do cuidado que lhes forem prestados ${ }^{7}$.

Este estudo tem como objetivo, des-
A satisfação no desenvolvimento das atividades laborais desenvolvidas pelos enfermeiros, está associada à manutenção da saúde do trabalhador e torna-se sinônimo, também, de qualidade de vida, pois contribui para o desenvolvimento de menores índices de adoecimento físico e mental crever, a partir da literatura publicada no período entre 2015 e 2020, a satisfação e insatisfação da equipe de enfermagem no contexto de trabalho em unidades de urgência e emergência.

\section{METODOLOGIA}

Trata-se de um estudo bibliográfico do tipo Revisão Integrativa (RI), com abordagem qualitativa. $\mathrm{O}$ desenvolvimento desta metodologia se dá em seis etapas: elaboração do tema e da pergunta norteadora; busca ou amostragem na literatura; coleta de dados; análise crítica dos estudos incluídos; discussão dos resultados; e apresentação da revisão integrativa ${ }^{8}$.

$\mathrm{Na}$ primeira etapa definiu-se como questão norteadora da pesquisa: Quais evidências científicas presentes na literatura nos últimos cinco anos, representam a satisfação da equipe de enfermagem no contexto de trabalho nas unidades de urgências e emergências? A questão foi elaborada com base na estratégia PICO, compreendida como (P) - População ("equipe de enfermagem"), (I) - Interesse ("satisfação da equipe de enfermagem") e (C)- Comparação ("Não se aplica"), (O) - desfecho ("Artigos científicos sobre satisfação da equipe de enfermagem no contexto da urgência e emergência").

Segunda etapa: Busca ou amostragem na literatura: foi realizada a seleção do material nos meses de setembro e outubro de 2020, com o apoio da questão norteadora e utilizando-se dos descritores cadastrados nos Descritores em Ciências da Saúde (DeCS): "Satisfação no TrabaIho"; "Enfermagem";" Enfermeiros"; "Ur- 
gência"; "Emergência", e os seus correspondentes em inglês: "Job satisfaction"; " Nursing"; " Nurses", "Urgency", "Emergency", utilizando-se os termos booleanos AND para tornar a busca mais eficaz.

A busca pelas publicações foi realizada, nas bases de dados MEDLINE, Literatura Latino-Americana e do Caribe em Ciências da Saúde (LILACS), Scientific Electronic Library Online (SciELO) e Biblioteca Virtual da Saúde (BVS). Como critérios de inclusão dos artigos especificou-se artigos originais, disponíveis na íntegra de forma gratuita; publicados nos últimos cinco anos (2015 a 2020) nos idiomas português, espanhol e inglês e relacionados com a questão de pesquisa. Foram excluídos capítulos de livros, resumos, textos incompletos, teses, dissertações, monografias, relatos técnicos e os que não atendiam ao objetivo do estudo. Foram utilizadas as recomendações propostas no guia PRISMA (Preferred Reporting Items for Systematic Reviews and Meta-Analysis) utilizando-se seu fluxograma para organização dos resultados de busca de artigos para Revisão Integrativa ${ }^{9}$. Conforme Figura 1.

A terceira etapa consistiu-se na coleta de dados: Nesta etapa, os artigos sele-

FIGURA 1: Preferred Reporting Items for Systematic Reviews and Meta-Analysis (PRISMA).

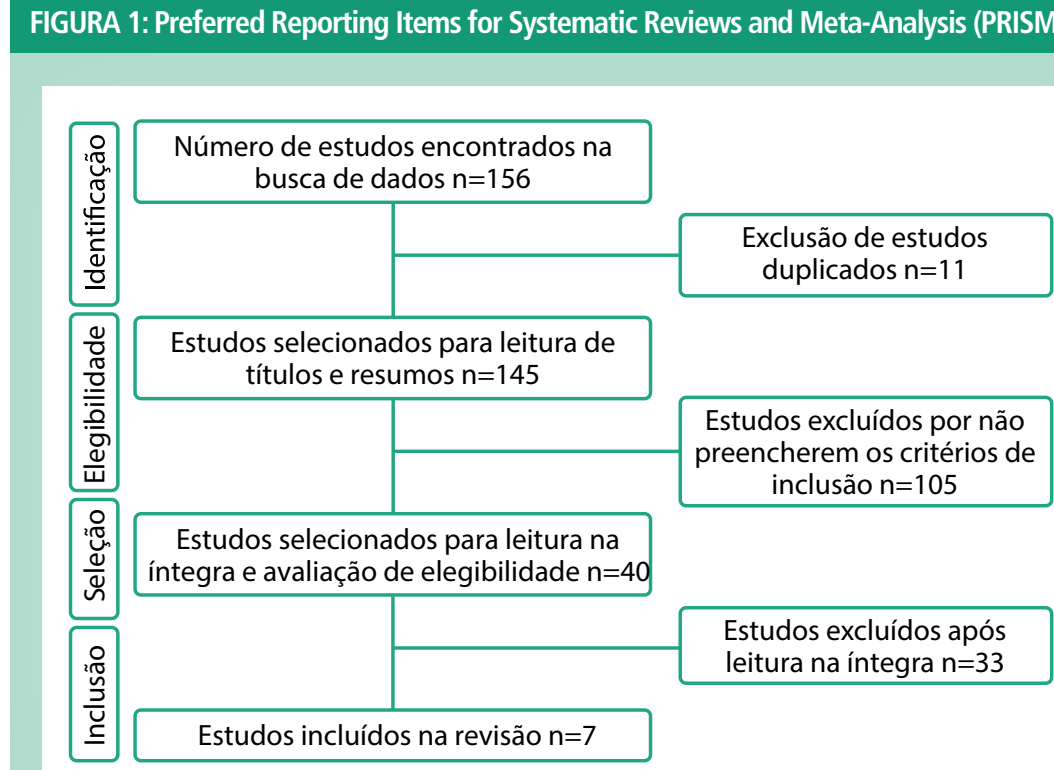

FONTE: Fluxograma representativo de busca e seleção artigos. Pará, 2020.

cionados foram lidos em sua íntegra e os dados de interesse registrados no formulário de coleta de dados ${ }^{10}$, abrangendo os seguintes itens: identificação dos artigos originais (procedência, título do artigo, autor (es), periódicos e consideração/ temática), instituição sede, tipo de publicação, características metodológicas do estudo, avaliação do rigor metodológico.

Quarta etapa: Análise crítica dos estudos incluídos: nesta etapa foi avaliada a significância metodológica dos estudos para classificação do nível de evidência, que foi de nível 4. Para análise das evidências foi aplicado o sistema de classificação de evidências caracterizado de forma hierárquica, dependendo da abordagem metodológica adotada, utilizando-se a hierarquia das evidências, em relação ao delineamento de pesquisa ${ }^{11}$. A análise dos dados se deu por meio da Análise de Conteúdo de Franco ${ }^{12}$, que destaca três etapas para sua aplicação: pré-análise; unidades de análise e definição de categorias.

A pré-análise, remete-se a organização do material já coletado de modo a organizar os "preâmbulos" com objetivo de desenvolver um plano de análise que meIhor se enquadre na pesquisa ${ }^{12}$. Nesta fase, organizou-se os artigos selecionados realizando sua leitura exaustiva e extraindo os dados de interesse para o formulário ${ }^{10}$. As informações contidas nos formulários foram sistematizadas em quadros sínteses a partir dos objetivos propostos para o estudo, verificando a pertinência das evidências e indicadores que apontaram algo comum entre os artigos.

Na segunda etapa - Unidades de análise, compreende-se a exploração do corpus, o qual, são escolhidas as unidades por critérios de codificação - um recorte; seleção de regras de contagem - enumeração; escolha de categorias - que possuem características em comuns e pôr fim a categorização em si, que promove a sistematização das informações de modo que, relacione as classes com os eventos ordenados $^{12}$. Nesta etapa as evidências levantadas na literatura, se constituíram de unidade de registro, observando-se os indicadores comuns, dando início ao processo de categorização. Na terceira fase foi elaborado duas categorias temáticas a partir das evidências levantadas nos artigos estudados, por meio das quais foram descritos e discutidos os resultados do estudo. Nas quinta e sexta etapas, foram então apresentados e discutidos os resultados com a elaboração da revisão e discutidas a luz do referencial teórico respondendo à questão de pesquisa e objetivo propostos no estudo.

\section{RESULTADOS}

Os artigos analisados tiveram como objetivo identificar e descrever os fatores de satisfação e insatisfação dos profissionais enfermeiros em unidades de urgência e emergência, assim como, relacionar variáveis demográficas a satisfação profissional nesse serviço e medir o nível de satisfação desses enfermeiros.

Os principais achados estão demonstrados no quadro 1. Em seguida os resultados encontram-se apresentados a partir de duas categorias temáticas: Fatores de satisfação no trabalho de enfermagem em urgência e emergência e Fatores de insa- 
tisfação no trabalho de enfermagem em urgência e emergência.

Categoria 1: Fatores de satisfação no trabalho de enfermagem em urgência e emergência
Nesta categoria temática observou-se que os trabalhadores de enfermagem em unidade de urgência e emergência sentem-se satisfeitos quando os requisitos do trabalho são bem explícitos, ou seja, quando a atividade laboral que execu- tam é bem compreendida em relação ao papel a ser realizado; quando ocorre boa interação entre a equipe de enfermagem, isto é, a existência de um bom relacionamento interpessoal, sendo o diálogo fundamental para produzir satisfação no

Quadro 1: Principais achados evidenciados nos artigos pesquisados. Pará, 2020.

\begin{tabular}{|c|c|c|}
\hline TÍTULO/IDIOMA/ANO & $\begin{array}{l}\text { BASE DE DADOS/ } \\
\text { REVISTA }\end{array}$ & RESULTADOS \\
\hline $\begin{array}{l}\text { LIDERANÇA E SATISFAÇÃO NO } \\
\text { TRABALHO NO CONTEXTO DO } \\
\text { SERVIÇO DE ATENDIMENTO MÓVEL } \\
\text { DE URGÊNCIA }{ }^{13} \text { Português } 2020\end{array}$ & $\begin{array}{l}\text { SCIELO Revista } \\
\text { Latino-Americana de } \\
\text { Enfermagem }\end{array}$ & $\begin{array}{l}\text { Constatou-se a autopercepção dos coordenadores em relação ao exercício da } \\
\text { Liderança Coaching apresentou correlação moderada com alguns domínios espe- } \\
\text { cíficos da satisfação no trabalho, entre eles, a "supervisão. }\end{array}$ \\
\hline $\begin{array}{l}\text { ÍNDICE DE SATISFAÇÃO DA EQUIPE } \\
\text { DE ENFERMAGEM ATUANTE EM UM } \\
\text { SERVIÇO DE PRONTO ATENDIMEN- } \\
\text { TO }{ }^{14} \text { Português } 2018\end{array}$ & $\begin{array}{l}\text { LILACS Enfermagem } \\
\text { Brasil }\end{array}$ & $\begin{array}{l}0 \text { domínio que mais contribuiu para a satisfação dos participantes deste estudo } \\
\text { foi Requisitos do trabalho classificado como muito bom. No que se refere ao do- } \\
\text { mínio que menos contribuiu com a satisfação profissional, observou-se o domínio } \\
\text { interação. Os domínios autonomia e remuneração também se fizeram importantes }\end{array}$ \\
\hline $\begin{array}{l}\text { PRAZER E SOFRIMENTO DE TRABA- } \\
\text { LHADORES DE ENFERMAGEM DE } \\
\text { UM PRONTO-SOCORRO }{ }^{15} \text { Português } \\
2018\end{array}$ & $\begin{array}{l}\text { SCIELO Texto Contexto } \\
\text { Enfermagem }\end{array}$ & $\begin{array}{l}\text { Os participantes mencionam que a satisfação no trabalho depende de outras } \\
\text { variáveis, entre elas, o relacionamento interpessoal. Apontaram descontentamento } \\
\text { com relação à grande quantidade de pacientes para cada profissional. A falta de } \\
\text { resolutividade para os problemas elencados pelos trabalhadores, por parte dos } \\
\text { gestores, constitui-se em um fator de estresse adicional. A falta de reconhecimen- } \\
\text { to no trabalho ou reforço de atitudes positivas no trabalho, a dor dos indivíduos } \\
\text { e a convivência diária com a morte também estão associados a situações que } \\
\text { geram sofrimento. }\end{array}$ \\
\hline $\begin{array}{l}\text { FATORES DE SATISFAÇÕES E } \\
\text { INSATISFAÇÕES NO TRABALHO DE } \\
\text { ENFERMEIROS }{ }^{16} \text { Português } 2020\end{array}$ & $\begin{array}{l}\text { SCIELO Revista de } \\
\text { enfermagem UFPE } \\
\quad \text { on line }\end{array}$ & $\begin{array}{l}\text { A falta de recursos materiais, equipamentos e a estrutura física inadequada da } \\
\text { instituição geraram insatisfação. Poucos materiais e muitos equipamentos sucate- } \\
\text { ados; há uma disputa para ver quem fica com os melhores materiais. Infraestrutu- } \\
\text { ra ruim para realizar cuidados e local para preparar medicação é péssimo. Fatores } \\
\text { que motivam a satisfação no trabalho do enfermeiro que contribuem para a } \\
\text { satisfação no trabalho: a capacitação, visto que o aprimoramento traz segurança } \\
\text { para realizar os cuidados. As reuniões que fazem mensalmente são momentos } \\
\text { que podem falar das dificuldades e potencialidades. }\end{array}$ \\
\hline $\begin{array}{l}\text { TRABALHO DE ENFERMAGEM EM } \\
\text { PRONTO SOCORRO PEDIÁTRICO: } \\
\text { ENTRE O PRAZER E O SOFRIMEN- } \\
\text { TO17 Português } 2019\end{array}$ & $\begin{array}{l}\text { LILLACS Cogitare } \\
\text { enfermagem }\end{array}$ & $\begin{array}{l}\text { Gostar de crianças e a interação e intervenção à família despontaram como ele- } \\
\text { mentos geradores de prazer. A possibilidade de realizar orientações de enferma- } \\
\text { gem e educação em saúde facilitada pelo fato de que a organização do trabalho } \\
\text { proporcionava tempo e tranquilidade para isso. Outra vivência geradora de prazer } \\
\text { no trabalho foi a recuperação da saúde da criança. Por fim, outro elemento mobi- } \\
\text { lizador do prazer no trabalho foi o reconhecimento }\end{array}$ \\
\hline $\begin{array}{l}\text { SATISFAÇÂO PROFISSIONAL DA } \\
\text { EQUIPE DE ENFERMAGEM X CON- } \\
\text { DIÇÕES E RELAÇÕES DE TRABALHO: } \\
\text { ESTUDO RELACIONAL' Português } \\
2015\end{array}$ & $\begin{array}{l}\text { SCIELO Texto Contexto } \\
\text { Enfermagem }\end{array}$ & $\begin{array}{l}\text { Este estudo apontou que condições laborais inapropriadas e inadequadas relações } \\
\text { no trabalho se associam à insatisfação laboral de profissionais de enfermagem } \\
\text { que atuam em unidades críticas (Centro Cirúrgico, Unidade de Terapia Intensiva } \\
\text { e Pronto Socorro). No que tange às condições de trabalho, sobressaíram àquelas } \\
\text { relacionadas ao tamanho da equipe, segurança e medicina do trabalho e acomo- } \\
\text { dações do setor. Quanto às relações de trabalho evidenciou-se insatisfação com a } \\
\text { distribuição das tarefas, estímulo profissional, valorização laboral e motivação. }\end{array}$ \\
\hline
\end{tabular}


trabalho e também para alcançarem eficácia no trabalho, como por exemplo, a estabilização do paciente e a recuperação dele; quando possuem autonomia e possuem remuneração que consideram satisfatória ${ }^{14,15}$.

Outro fator apontado como de satisfação no trabalho está relacionado ao reconhecimento pela qualidade do trabaIho que é realizado, no sentido de compreender todo esforço que foi feito pela equipe de enfermagem para a prática laboral; além disso, a boa evolução clínica do paciente e sua recuperação são fatores que geram satisfação pelos resultados positivos alcançados ${ }^{15}$.

A capacitação, educação continuada, que promove o aprimoramento do conhecimento foi também apontado como fator de satisfação no trabalho de urgência e emergência, visto que contribui para sentirem-se seguros na realização dos cuidados e no exercício de suas funções; outro fator de satisfação é a oportunidade de falarem, de serem escutados em suas dificuldades e sugestões, nesse caso apontam a importância das reuniões mensais no serviço, momento que podem se expressar, que são elogiados e até melhoram sua relação interpessoal. Além do que, a educação em saúde e a recuperação do paciente são apontados como fatores geradores de satisfação no trabalho ${ }^{17}$.

Um estudo realizado com enfermeiros que trabalham em serviço de urgência aéreo demonstra que eles consideram esse trabalho diferente, interessante e, portanto, leva a realização profissional, a autorrealização como fator de satisfação no trabalho, além de contribuírem de forma efetiva para a reabilitação da pessoa $^{18}$. Outro aspecto evidenciado, foi em relação ao tipo de liderança, demonstrando que a liderança coaching leva a satisfação no trabalho no domínio supervisão e comunicação ${ }^{13}$.

Categoria 2: Fatores de insatisfação no trabalho de enfermagem em urgência e emergência
$\mathrm{Na}$ categoria 2, observou-se como fatores que geram insatisfação no trabalho, a falta de interação entre a equipe de enfermagem; falta de reconhecimento profissional; falta de autonomia e apoio por parte dos supervisores e chefes; o número grande pacientes por profissional que leva ao cansaço e esgotamento físico pela sobrecarga de trabalho; a distribuição de tarefas; falta de resolutividade para os problemas levados aos dirigentes; alta exigência do trabalho; falta de reconhecimento no trabalho principalmente pela chefia imediata e gestão institucional $\left.\right|^{1,14-16}$.

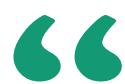

A remuneração está relacionada a satisfação, visto que, proporciona a redução de profissionais fadigados com vários vínculos empregatícios, jornada de trabalho satisfatória e um salário ideal

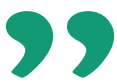

As condições de trabalho, também foram apontadas como fatores de insatisfação, pois os profissionais se deparam com a falta de recursos materiais, equipamentos e estrutura física inadequada para realizar cuidados, preparo de medicação, fazer higiene dos pacientes, dificultando a humanização do cuidado prestado ${ }^{15,16}$.
Outro fator que gera sofrimento e, insatisfação com o trabalho em urgência e emergência está relacionado com os sentimentos vivenciados pelos profissionais de enfermagem ao lidar com a dor, o sofrimento das pessoas e a morte quase sempre diária, relatam que esse sofrimento é maior quando se trata de crianças e jovens; lidar com o estresse dos pais diante do quadro grave de seu filho e atender crianças vítimas de violência, também são fatores que levam a insatisfação ${ }^{15,17}$.

\section{DISCUSSÃO}

A categoria 1 aponta como fatores determinantes da satisfação da equipe de enfermagem em urgência e emergência: requisitos do trabalho; boa interação interpessoal; autonomia; remuneração; reconhecimento pelo trabalho; recuperação do paciente; educação continuada; serem escutados; serem reconhecidos e valorizados; autorrealização com o trabalho; liderança coaching. Essa realidade é corroborada por outros autores, pois o trabalho que, pode causar satisfação pessoal é aquele que permite a aquisição salarial ideal, trabalhar na área de atuação desejada e ainda ter recursos disponíveis para desenvolvimento de atividades de trabalho com êxito. A remuneração está relacionada a satisfação, visto que, proporciona a redução de profissionais fadigados com vários vínculos empregatícios, jornada de trabalho satisfatória e um salário ideal ${ }^{19}$.

Além disso, o reconhecimento do paciente e/ou acompanhante sobre o cuidado prestado exercido pela equipe, reforçam o reconhecimento e prazer durante a prática laboral desses profissionais. Contudo, esse sentimento de realização profissional só é possível, mediante a liberdade e autonomia de exercer as atribuições profissionais a que estes profissionais são regidos ${ }^{20}$.

A educação continuada no ambiente de trabalho de urgência e emergência é uma estratégia desafiadora tanto para a 
equipe, quanto para gestão e as propostas e metodologias devem ser estudadas com o objetivo de alcançar os profissionais que estão inseridos nessa realidade e aplicar durante a assistência ${ }^{21}$. Por fim, ressalta-se a questão de liderança, pois o enfermeiro é capacitado para liderar a equipe de enfermagem, porém, uma boa liderança precisa estabelecer diálogo com a equipe, de modo articulado deixando clareza em seus objetivos e em prol do paciente assistido ${ }^{22}$.

$\mathrm{Na}$ categoria 2 evidenciou-se fatores de insatisfação como: falta de interação, de reconhecimento profissional, de autonomia, de apoio por parte da chefia. Ainda, o grande número de pacientes por profissional, falta de resolutividade para problemas do serviço, condições de trabalho inadequadas e o sofrimento gerado pela natureza do serviço de lidar com o sofrimento, com a morte, com o estresse de familiares frente a gravidade de seus pacientes. Esses aspectos são corroborados por autores que enfatizam a sobrecarga de trabalho como fator de insatisfação no ambiente laboral, pois os profissionais deparam-se com um cenário de falta de recursos humanos comparados a demanda de pessoas que solicitam o serviço de saúde, provocando não somente na insatisfação dos profissionais envolvidos como também aumento do nível de estresse nesses trabalhadores ${ }^{23}$.

Por meio do trabalho as pessoas buscam alcançar seus objetivos e aspirações. É uma forma de obter qualidade de vida já que esta está diretamente relacionada a satisfação individual entre as diversas esferas da vida humana, seja ela pessoal ou profissional. Assim, o trabalho também pode ser responsável por gerar fatores de estresse e desgaste e ocasionar desequilíbrios na saúde do trabalhador ${ }^{24}$.

A sobrecarga de trabalho vivenciada por profissionais de enfermagem é um fator de grande relevância no que tange a qualidade da assistência prestada, e nas inter-relações entre a tríade enfermeiro-equipe-usuário, sendo um forte agente estressor para esses trabalhadores ${ }^{25}$. $\mathrm{O}$ estresse devido à falta de recursos materiais e deficiência do espaço físico para o desempenho do trabalho dos profissionais de saúde, acarreta a insatisfação desses trabalhadores, e pode influenciar na eficácia do trabalho desempenhado e na falta de resolutividade dos desafios enfrentados diariamente ${ }^{26}$. Ressalta-se, ainda, que um dos quesitos que causam maior insatisfação em relações as condições de trabalho estão relacionadas à desvalorização e falta de reconhecimento no ambiente de trabaIho, confirmado pela falta de recompensa financeira e falta de expectativa de ascensão na carreira ${ }^{27}$.

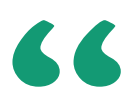

Na categoria 2 evidenciouse fatores de insatisfação como: falta de interação, de reconhecimento profissional, de autonomia, de apoio por parte da chefia.

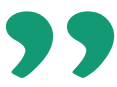

Esses resultados vão ao encontro de outros estudos quanto ao ambiente de trabalho poder se tornar um lugar gerador de experiências e vivencias positivas ou negativas na perspectiva de cada trabalhador e que pode provocar prejuízos a saúde do profissional, assim como, influenciar na percepção que cada profissional tem de sua profissão ${ }^{21}$.

\section{CONCLUSÃO}

O estudo evidenciou que, são fatores geradores de satisfação para a equipe de enfermagem no contexto de trabalho em urgência emergência: a presença de requisitos do trabalho; boa interação interpessoal; autonomia; remuneração; reconhecimento pelo trabalho; recuperação do paciente; educação continuada; ser ouvido, reconhecido e valorizado; autorrealização com o trabalho e a liderança coaching. Já como fatores de insatisfação, encontram-se: falta de interação, de reconhecimento profissional, de autonomia, de apoio por parte da chefia, falta de resolutividade para problemas do serviço, condições de trabalho inadequadas, como a sobrecarga, e o sofrimento gerado pela natureza do serviço de lidar com o sofrimento, com a morte, com o estresse de familiares frente a gravidade de seus pacientes.

Nesse sentido, conclui-se que as condições de estrutura física, recursos materiais, relações interpessoais e educação permanente, são fatores que se adequados, contribuem para a satisfação no trabalho e humanização dos trabalhadores e dos pacientes atendidos nessas unidades. No entanto, o estudo demonstra que nos últimos cinco anos pouco se publicou em relação ao tema, havendo necessidade de impulsionar pesquisas sobre o tema, visto os resultados aqui evidenciados.

É premente, ainda, a necessidade de estudos que abordem a temática explorando temas como a liderança coaching e o protagonismo da enfermagem nessa área que requer atenção pela complexidade e natureza do cuidado que nela é prestado. Além do que, este estudo pode contribuir como base para políticas organizacionais sobre o trabalho de enfermagem em unidades de Urgência e 
Emergência, visto que traz evidências do que essa equipe percebe para sua satis- fação e qualidade da assistência ofertada em um setor em que, esses profissionais lidam diariamente com situações limites de estresse, sofrimento, dor e morte.

\section{Referências}

1. Wisniewski D, Silva ES, Évora YDM, Matsuda LM. Satisfação profissional da equipe de enfermagem $x$ Condições e relações de trabalho: estudo relacional. Texto Context - Enferm [Internet]. 2015 Sep;24(3):850-8. Available from: http://www. scielo.br/scielo.php?script=sci_arttext\&pid=S0104-07072015000300850\& $\mathrm{I}$ $\mathrm{ng}=\mathrm{en} \&$ tlng=en

2. Correia MMSV. Satisfação profissional dos enfermeiros.115f. Dissertação (Mestrado em Direção e Chefia de Serviços de Enfermagem). Escola superior de enfermagem do Porto, Porto, Portugal. 2016. Available from: https://comum. rcaap.pt/handle/10400.26/18161

3. Forte ECN, Pires DEP. Enfermeiras na atenção básica: entre a satisfação e a insatisfação no trabalho. Trab Educ e Saúde [Internet]. 2017 Dec;15(3):709-

24. Available from: http://www.scielo.br/scielo.php?script=sci_arttext\&pid=\$1981-77462017000300709\&lng=pt\&tlng=pt

4. Brasil. Ministério da Saúde. Gabinete do Ministro. Comissão Intergestora Tripartite. Portaria no 2048, de 05 de novembro de 2002: Regulamento Técnico dos Sistemas Estaduais de Urgência e Emergência. Diário Oficial da União. 2002 6 nov. Available from: https://bvsms.saude.gov.br/bvs/saudelegis/gm/2002/ prt2048 05 11 2002.html

5. Acosta AM, Marques GQ, Levandovski PF, Peralta JP, Lima MADS. User satisfaction regarding nursing care at emergency services: an integrative review. REME Rev Min Enferm [Internet]. 2016;20. Available from: http://www.gnresearch.org/doi/10.5935/1415-2762.20160008

6. Cardoso RFL, Oliveira LC, Parente JS. Dificuldades vivenciadas pelo enfermeiro assistencial nas unidades de urgência e emergência: uma revisão integrativa. Res Soc Dev [Internet]. 2021 Feb 16;10(2):e29510212487. Available from: https://rsdjournal.org/index.php/rsd/article/view/12487

7. Santos MA, Sardinha AHL, Santos LN dos. Satisfação dos usuários com os cuidados dos enfermeiros. Rev Gaúcha Enferm [Internet]. 2017;38(1). Available from: http://www.scielo.br/scielo.php?script=sci_arttext\&pid=S1983-14472017000100401\&lng=pt\&tlng=pt

8. Koopmans FF, Daher DV, Acioli S, Sabóia VM, Ribeiro CRB, Silva CSSL. Living on the streets: An integrative review about the care for homeless people. Rev Bras Enferm [Internet]. 2019 Feb;72(1):211-20. Available from: http://www.scielo.br/ scielo.php?script=sci_arttext\&pid=S0034-71672019000100211\&tlng=en 9. Moher D, Shamseer L, Clarke M, Ghersi D, LiberatiA, Petticrew M, et al. Preferred reporting items for systematic review and meta-analysis protocols (PRISMA-P) 2015 statement. Syst Rev [Internet]. 2015 Dec 1;4(1):1. Available from: https:// systematicreviewsjournal.biomedcentral.com/articles/10.1186/2046-4053-4-1 10. Ursi ES, Gavão CM. Prevenção de lesões de pele no perioperatório: revisão integrativa da literatura. Rev Lat Am Enfermagem [Internet]. $2006 \mathrm{Feb}$ [cited 2019 Apr 20]:14(1):124-31. Available from: http://www.scielo.br/scielo. php?script=sci_arttext\&pid=S0104-11692006000100017\&lng=pt\&tlng=pt

11. Ercole FF, Melo LS de, Alcoforado CLGC. Integrative review versus systematic review. Reme Rev Min Enferm [Internet]. 2014;18(1). Available from: http:// www.gnresearch.org/doi/10.5935/1415-2762.20140001

12. Franco MLPB. Análise de conteúdo. Brasília: Líber Livro; 2005.

13. Moura AA, Bernardes A, Balsanelli AP, Dessotte CAM, Gabriel CS, Zanetti ACB. Leadership and job satisfaction in the Mobile Emergency Care Service context. Rev Lat Am Enfermagem [Internet]. 2020;28. Available from: http://www. scielo.br/scielo.php?script=sci_arttext\&pid=S0104-11692020000100323\&t|$\mathrm{ng}=\mathrm{en}$

14. Oliveira VNDS, Hertel VL, Silva PR. Índice de satisfação da equipe de enfermagem atuante em um serviço de pronto atendimento. Enferm Bras [Internet]. 2018 Sep 28;17(4):370. Available from: http://portalatlanticaeditora.com.br/index.php/enfermagembrasil/article/view/2548

15. Miorin JD, Camponogara S, Pinno C, Beck CLC, Costa V, Freitas EO. Prazer e sofrimento de trabalhadores de enfermagem de um pronto-socorro. Texto Context - Enferm [Internet]. 2018 May 3;27(2). Available from: http://www.scielo. br/scielo.php?script=sci_arttext\&pid=S0104-07072018000200305\&lng=pt\&t|- $\mathrm{ng}=\mathrm{pt}$

16. Marques CR, Ribeiro BMSS, Martins JT, Dias HG, Dalri RCDMB, Bernardes $M L G$, et al. Fatores de satisfações e insatisfações no trabalho de enfermeiros. Rev Enferm UFPE line [Internet]. 2020 Jun 8;14. Available from: https://periodicos. ufpe.br/revistas/revistaenfermagem/article/view/244966

17. Lamb FA, Beck CLC, Coelho APF, Vasconcelos RO. Trabalho de enfermagem em pronto socorro pediátrico: entre o prazer e o sofrimento. Cogitare Enferm [Internet]. 2019 May 20;24. Available from: https://revistas.ufpr.br/cogitare/article/view/59396

18. Lima EWM, Mota MHLV, Rocha FCVV, Madeira MZA, Oliveira ADS. Satisfaction of nurses in service mobile service emergency air. Rev Enferm da UFPI [Internet]. 2015 Dec 1;4(4):49. Available from: http://www.ojs.ufpi.br/index.php/ reufpi/article/view/3504

19. Cordeiro MG, Torres ARA, Rocha FAA, Costa FBF, Branco JGO. Satisfação profissional de enfermeiros em uma unidade de emergência. Nursing (São Paulo). 2019;22(249):2604-9. Available from: http://revistas.mpmcomunicacao. com.br/index.php/revistanursing/article/view/246

20. Alves SR, Santos RP, Yamaguchi MU. Enfermagem em serviços de saúde mental: percepção sobre satisfação profissional e condições de trabalho. Rev Enferm do Centro-Oeste Min [Internet]. 2018 Mar 20;8. Available from: http:// seer.ufsj.edu.br/index.php/recom/article/view/1852

21. Silva DP.A perspectiva do enfermeiro frente a sua valorização profissional e social. In:Anais do 170 Congresso Nacional de Iniciação Científica CONIC/SEMESP, Centro Universitário Ítalo Brasileiro, 2017;5. Available from: http://conic-semesp. org.br/anais/anais-conic. php?ano=2017\&idautor $=42135129836 \&$ act=pesquisar

22. Ferro D, Zacharias FCM, Fabriz LA, Schonholzer TE, Valente SH, Barbosa SM, et al. Absenteísmo na equipe de enfermagem em serviços de emergência: implicações na assistência. Acta Paul Enferm [Internet]. 2018 Jul;31(4):399408. Available from: http://www.scielo.br/scielo.php?script=sci_arttext\&pid=S0103-21002018000400399\&lng=pt\&tlng=pt

23. Trettene AS, Ferreira JAF, Mutro MEG, Tabaquim M de LM, Razera APR. Estresse em profissionais de enfermagem atuantes em Unidades de Pronto Atendimento. Bol - Acad Paul Psicol. 2016;36(91):243-61. Available from: http://pepsic. bvsalud.org/scielo.php?script=sci_abstract\&pid=\$1415-711X2016000200002

24. Nogueira da Silva PL, Leite Pereira I, Fonseca Coelho Galvão AP, Lopes de Figueiredo M, Carolina dos Reis Alves C dos RA, Gomes e Martins A. Fatores intervenientes na qualidade de vida de trabalhadores de enfermagem em jornada noturna. Nursing (São Paulo) [Internet]. 2021 Jan 4;24(272):5172-97. Available from: http://www.revistas.mpmcomunicacao.com.br/index.php/revistanursing/ article/view/1118

25. Duarte VRC. A sobrecarga de trabalho na atuação do enfermeiro na estratégia de saúde da família. 34f. Monografia (Especialização em Atenção Básica em Saúde da Família). Universidade Federal de Minas Gerais, Conselheiro Lafaiete, Brasil. 2013. Available from: https://www.nescon.medicina.ufmg.br/bibliotecal registro/A_sobrecarga_de_trabalho_na_atuacao_do_enfermeiro_na_Estrategia_de_Saude_da_Familia/461

26. Amaral APS, Longuiniere ACFD La, Santos JNM de 0, Vilela ABA, Vieira SNS, Sanches $\mathrm{G}$ de JC. Occupational Stress: the Exposure of an Emergency Unit Nursing Team. Rev Pesqui Cuid é Fundam Online [Internet]. 2019 Jan 21;11(2):455. Available from: http://seer.unirio.br/index.php/cuidadofundamental/article/ view/6386

27. Pinho CM, Fonseca BRL da, Cabral LM da S, Maia T de S, Cipriano AA de S, Silva SRC da, et al. Aspectos associados ao estresse em enfermeiros que atuam em serviços de urgência e emergência. Rev Enferm Digit Cuid e Promoção da Saúde [Internet]. 2020;5(1). Available from: http://www.redcps.com.br/detalhes/78/aspectos-associados-ao-estresse-em-enfermeiros-que-atuam-em-servicos-de-urgencia-e-emergencia 